\title{
SHH medulloblastoma in a young adult with a TCF4 germline pathogenic variation
}

Maud Blanluet ${ }^{1}$, Julien Masliah-Planchon ${ }^{1,2}$, Irina Giurgea ${ }^{3,4}$, Franck Bielle ${ }^{5}$, Elodie Girard ${ }^{6}$, Mamy Andrianteranagna ${ }^{2}$, Stéphane Clemenceau ${ }^{7}$, Christine Bourneix $^{1}$, Lydie Burglen ${ }^{8}$, Diane Doummar $^{9}$, Audrey Rapinat ${ }^{10}$, Badreddine Mohand Oumoussa ${ }^{11}$, Olivier Ayrault ${ }^{12,13}$, Celio Pouponnot ${ }^{12,13}$, David Gentien ${ }^{10}$, Gaëlle Pierron ${ }^{1,2}$, Olivier Delattre ${ }^{1,2,14}$, François Doz ${ }^{2,15}$, Franck Bourdeaut ${ }^{2,14}$

$1 \quad$ Unité de Génétique Somatique, Institut Curie, Paris, France

2 SIREDO Oncology Centre (Care, innovation, research in pediatric, adolescent and young adults oncology), Institut Curie, Paris, France

3 UF de Génétique Moléculaire, Hôpital Armand Trousseau, APHP, Paris, France

$4 \quad$ Sorbonne Université, INSERM UMR S933, Paris, France.

5 Departement de neuropathologie, groupe hospitalier Pitié-Salpêtrière, AP-HP, Paris, France.

6 INSERM U900, Institut Curie, Paris, France.

7 Neurochirurgie, GH Pitie-Salpêtrière-Charles Foix, APHP, Paris, France.

8 Centre de Référence Maladies Rares «Malformations et Maladies Congénitales du Cervelet ", et Département de Génétique, Hôpital Armand Trousseau, GHUEP, APHP, Paris, France

9 Département de neuropédiatrie, Hôpital Armand Trousseau, APHP, Paris, France

10 Institut Curie, PSL Research University, Translational Research Department, Genomics platform, Paris, F-75248 France

11 Sorbonne Université, Inserm, UMS Omique, Plateforme Post-génomique de la PitiéSalpêtrière, P3S, F-75013, Paris, France

12 Université Paris Sud, Université Paris-Saclay, CNRS UMR 3347, INSERM U1021, Orsay, France.

13 Institut Curie, PSL Research University, CNRS UMR, INSERM, Orsay, France

14 Inserm U830, PSL Université, Institut Curie, Paris, France

15 Université Paris Descartes, Sorbonne Paris Cité, Paris, France

Correspondance to: Dr Franck Bourdeaut.

franck.bourdeaut@curie.fr

Pediatric Oncology Departement

26, rue d'UIm 75248 PARIS CEDEX 05 France

fax number: +33153104005 
Key words:

Medulloblastoma, adult, Pitt-Hopkins, TCF4, predisposition

No conflict of interest 
Pitt-Hopkins syndrome (PTHS, MIM \#610954) is a rare neurodevelopmental disease due to heterozygous loss of function variants in the TCF4 gene (transcription factor 4, MIM \#602272) [1]. TCF4 encodes a basic helix-loop-helix (bHLH) transcription factor which is highly expressed in the nervous system during early development and is involved in cellular proliferation and differentiation. To date, approxi-mately 200 PTHS patients have been reported since the first clinical description in 1978 [1, 11, 12]. The limited number of cases described and their early age precludes establishing a comprehensive phenotype, especially regarding cancer pre-disposition. Here we report the case of a 27year-old woman affected by PTHS who developed a medulloblastoma (MB).

The PTHS patient, previously reported (P12) [11], har-bored a typical facial gestalt, hypotonia, hyperventilation, had delayed walking, and never acquired language. The PTHS diagnosis was confirmed by identification of the heterozygous de novo pathogenic frameshift variant c.1241del, p.(Gly414Valfs*48) in the TCF4 gene (NM_001083962.1). The patient developed a posterior fossa tumor at the age of 27 years arising from the right cerebellar hemisphere (Fig. 1a). The tumor has been macroscopically completely resected and the anatomopathological examination established a diagnosis of classic MB with $\mathrm{SHH}$ immunohistochemical profile (filamin+, GAB1+, Supplementary Fig. 1). The patient was then treated using standard dose of craniospinal radiotherapy without post-radiation chemotherapy. Array-CGH performed on the tumor DNA revealed multiple copy number alterations including GLI2 amplification, but no MYC/MYCN amplification and no loss of heterozygosity at the TCF4 locus (Fig. 1d). Nanostring molecular subgrouping [8] and RNAseq analysis unambiguously confirmed the SHH group (Fig. 1b). Nonetheless, based on the DKFZ classifier tool (https ://www.molec ularneurop athol ogy.org) [2], the 
methylation analysis did not confidently match any CNS tumor class. The closest entity was SHH MB followed by IDH mutated gliomas (Supplementary Fig. 2; GSE126545). Sequencing of a custom cancer genes panel on tumor DNA identified the pathogenic variants C.-124C $>\mathrm{T}$ in the TERT promoter (NM_198253.2) and c.514A > G, p.(Arg172Gly) in the IDH2 gene (NM_002168.3) (Fig. 1c; Supplementary Fig. 2). The TERTpromoter variation occurred in a hot spot position which is recurrent in adult SHH MB [9]. No TCF4 second alteration was identified in the tumor sample, neither at the genomic nor at the transcript level (GSE126545).

A recently published large-scale analysis of germline pathogenic variants associated with $\mathrm{MB}$ revealed that $\mathrm{SHH} M B$ is the most likely to arise in the context of a genetic predisposition [10]. Germline PTCH1 and SUFU pathogenic variants occur in approximately $10 \%$ of $\mathrm{SHH} \mathrm{MB}$ in infants and young children, while recessive diseases affecting DNA damage repair (i.e., Fanconi Anemia with bi-allelic inactivation of PALB2 or BRCA2) are also associated with pediatric SHH MB. SHH MB in older patients is much less frequently associated with germline pathogenic variants, apart from rare TP53 pathogenic variants in Li-Fraumeni syndrome. Thus, no gene is yet known to specifically predispose to adultonset SHH MB. The case we report here raises the the hypothesis that PTHS due to germline TCF4 pathogenic variants confers increased susceptibility to adult-onset SHH MB. However, a random association of those two rare diseases needs to be carefully ruled out in future studies.

Indeed, to date no MB has been described in PTHS patients. Nevertheless, scarcely more than 200 PTHS cases have been reported worldwide. In one of the biggest cohorts described, including 101 PTHS patients [12], only two were older than 25 years old. Hence, 
the susceptibility to adult-onset MB in PTHS is difficult to accurately estimate because of the small number of identified PTHS patients that have reached adulthood, which could mask a slightly elevated age-related risk.

Remarkably, in a large-scale genome wide analysis of $491 \mathrm{MB}$, eleven SHH MBs and one group $4 \mathrm{MB}$ were reported to show somatic TCF4 variants [7]. TCF4 variants in SHH MB were mainly truncating variants (8/11) in favor of a role of TCF4 loss in SHH MB. Of note, in line with our case, all but one was heterozygous, with no second hit, strongly suggesting a haploinsufficiency mechanism for TCF4 in promoting MB. Thus, TCF4 may act similar to other tumor suppressors and cancer-predisposing genes, for which heterozygous truncating mutations leading to haploinsufficiency are now recurrently reported (updated review in [4]). Of note, all pathogenic TCF4 variants were described in adult-onset tumors [7], strongly suggesting an age-related oncogenic effect of TCF4 pathogenic variations. In line with previous studies investigating the role of CREBBP loss of function in MB according to developmental stages [6], Hell-wig et al. describe in this issue of Acta Neuropathologica that TCF4 abrogation increases cell proliferation only on post-natal granule cell progenitors, providing experimental evidence for an age-related oncogenic effect of TCF4pathogenic variations [3]. Altogether, these results suggest a bivalent effect of TCF4 pathogenic variations, i.e., inducing a developmental disorder in the developing brain and an increased risk of malignant transformation in the adult cerebellum. This could explain the low known incidence of MB in PTHS patients, and fits with the late-onset in this case report.

Of note, all but one of the aforementioned TCF4 mutated MB belonged to the SHH group [7], a finding consistent with previous results identifying TCF4 recurrent pathogenic 
variations as one of the most frequent somatic events in adult SHH MB [5]. The strikingly unbalanced distribution of TCF4 alterations among the four MB groups strongly suggests a specific cooperation between constitutive activation of the SHH pathway and TCF4 loss of function. Yet how TCF4 pathogenic variations interact and synergize with the SHH pathway remains to be more deeply investigated.

We postulate that TCF4 germline alterations confer increased susceptibility to lateonset SHH MB. The aging of patients affected by PTHS should bring further insights into this hypothesis and would strengthen the need for further exploration of the potential synergy between TCF4 and the SHH pathway in MB. 


\section{References}

1. Amiel J, Rio M, de Pontual L, Redon R, Malan V, Boddaert N et al (2007) Mutations in TCF4, encoding a class I basic helix-loop-helix transcription factor, are responsible for Pitt-Hopkins syndrome, a severe epileptic encephalopathy associated with autonomic dysfunction. Am J Hum Genet 80:988993. https ://doi.org/10.1086/51558 2

2. Capper D, Jones DTW, Sill M, Hovestadt V, Schrimpf D, Sturm D et al (2018) DNA methylation-based classification of cen-tral nervous system tumours. Nature 555:469-474. https ://doi.org/10.1038/natur e2600 0

3. Hellwig M, Laufer MC, Bockmayr M, Spohn M, Merk DJ, Har-rison L et al (2019) TCF4 (E2-2) harbors tumor suppressive functions in SHH medulloblastoma. Acta Neuropathol. https ://doi.org/10.1007/s0040 1-019-01982 -5

4. Inoue K, Fry EA (2017) Haploinsufficient tumor suppressor genes. Adv Med Biol 118:83-122

5. Kool M, Jones DTW, Jäger N, Northcott PA, Pugh TJ, Hovestadt V et al (2014) Genome sequencing of $\mathrm{SHH}$ medul-loblastoma predicts genotype-related response to smoothened inhibition. Cancer Cell 25:393-405. https ://doi.org/10.1016/j.ccr.2014.02.004

6. Merk DJ, Ohli J, Merk ND, Thatikonda V, Morrissy S, Schoof M et al (2018) Opposing effects of CREBBP mutations gov-ern the phenotype of Rubinstein-Taybi syndrome and adult SHH medulloblastoma. Dev Cell 44:709-724.e6. https ://doi.org/10.1016/j.devce I.2018.02.012

7. Northcott PA, Buchhalter I, Morrissy AS, Hovestadt V, Weis-chenfeldt J, Ehrenberger T et al (2017) The whole-genome land-scape of medulloblastoma subtypes. Nature 547:311-317. https://doi.org/10.1038/natur e2297 3

8. Northcott PA, Shih DJH, Remke M, Cho Y-J, Kool M, Hawkins C et al (2012) Rapid, reliable, and reproducible molecular sub-grouping of clinical medulloblastoma samples. Acta Neuropathol (Berl) 123:615-626. https ://doi.org/10.1007/s0040 1-011-0899-7

9. Remke M, Ramaswamy V, Peacock J, Shih DJH, Koelsche C, Northcott PA et al (2013) TERT promoter mutations are highly recurrent in SHH subgroup medulloblastoma. Acta Neuro-pathol (Berl) 126:917-929. https ://doi.org/10.1007/s00401-013-1198-2

10. Waszak SM, Northcott PA, Buchhalter I, Robinson GW, Sutter C, Groebner S et al (2018) Spectrum and prevalence of genetic predisposition in medulloblastoma: a retrospective genetic study and prospective validation in a clinical trial cohort. Lancet Oncol 19:785-798. https ://doi.org/10.1016/S1470-2045(18)30242 -0

11. Whalen S, Héron D, Gaillon T, Moldovan O, Rossi M, Devil-lard F et al (2012) Novel comprehensive diagnostic strategy in Pitt-Hopkins syndrome: clinical score and further delineation of the TCF4 mutational spectrum. Hum Mutat 33:64-72. https://doi.org/10.1002/humu.21639

12. de Winter CF, Baas M, Bijlsma EK, van Heukelingen J, Rout-ledge S, Hennekam RCM (2016) Phenotype and natural history in 101 individuals with Pitt-Hopkins syndrome through an inter-net questionnaire system. Orphanet J Rare Dis 11:37. https ://doi.org/10.1186/s1302 3-016-0422-2 


\section{Legends}

Figure 1. Imaging and molecular features of the tumor. (a) Brain MRI axial T1 with gadolinium injection revealing a tumor in the right cerebellar hemisphere. (b) Hierarchical clustering of $113 \mathrm{MB}$ from Curie dataset, based on the Nanostring signature made of 22 medulloblastoma subgroup-specific genes: the tumor reported in this correspondence is indicated by the arrow and orange dotted line box. Analysis of tumor DNA by the Curie Institute custom cancer panel assessing known cancer-related genes; the two relevant mutated genes in this panel are indicated in bubbles; bubbles sizes are correlated to sequencing depth at variant positions and the read count for alternative variant related to the reference nucleotide is specified in brackets. The allele ratio is specified in the $x$ axis. (c) The TCF4 pathogenic variation assessed by NGS is added in blue. (d) Array-CGH performed on tumor DNA revealed several copy number alterations including GLI2 and VEGFC amplifications but no loss of heterozygosity at the TCF4 locus (red arrow; 18q12.2).

Supplementary Figure 1. Histopathological features of the tumor. (a) Hematoxylin and eosin staining showed a histological aspect of classic medulloblastoma with necrosis. (b) Immnunostainings showed a diffusely high Ki67 proliferation index, (c) a maintained expression of SMARCB1/INI1, and (d) a mild positivity of P53. (e) Reticulin staining showed no reticulinic network between tumor cells. (f) Immunostainings showed a membrane staining for Beta-catenin, and (g) a strong positivity for Filamin-1 and (h) GAB1 favoring the genetic group of SHH-activated medulloblastomas.

Supplementary Figure 2. Methylation and genomic tumor data. (a) t-distributed Stochastic Neighbor Embedding (t-SNE) analysis using DNA methylation data (generated using 
HumanMethylation450K array type) of glioblastoma (GBM) and medulloblastoma (MB) samples published in Capper et al. (2018) [2] and DNA methylation data of our case (generated using HumanMethylationEPIC array type) named MB699. (b) MNP2.0-based DNA methylation class prediction calibrated scores for our case (DKFZ classifier version: 11b4). The other scores (data not shown) are inferior to 0.01. (c) Analysis of tumor DNA of our case by the Curie Institute custom cancer panel assessing known cancer-related genes ( 8 alterations), compared with the proportion of relevant tumor DNA alterations of these genes for all SHH MB (SHH MB column) and TCF4 mutated SHH MB (TCF4 mutated SHH MB column) samples published by Northcott et al. (2017) [6]. 
a

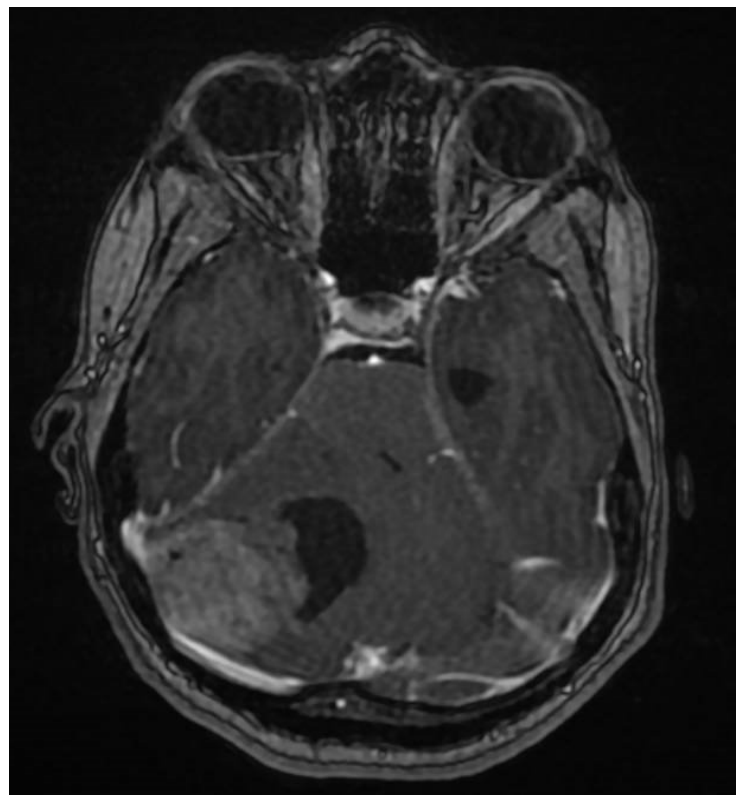

C

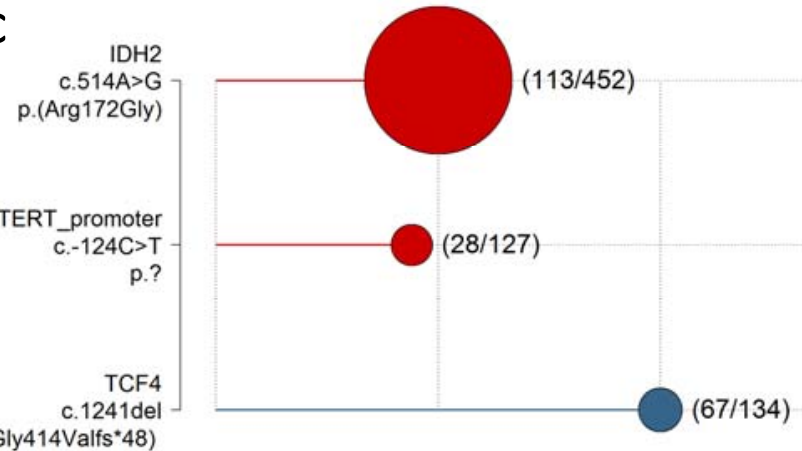

C.1241del
p.(Gly414Valfs*48)

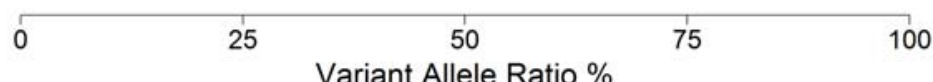

d

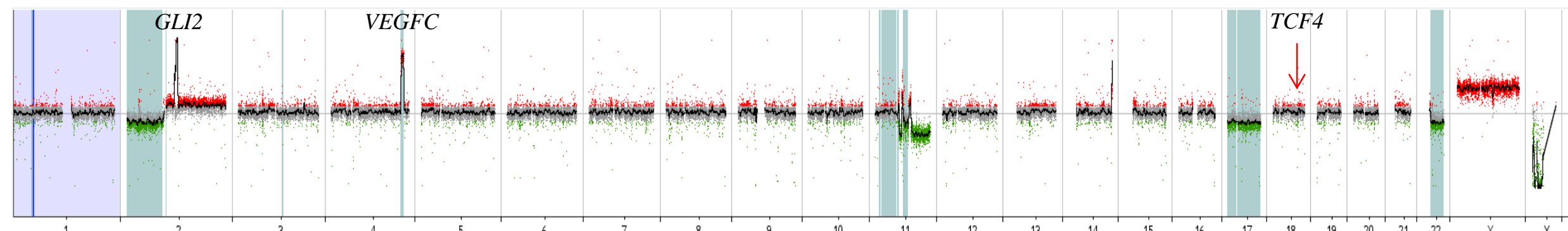

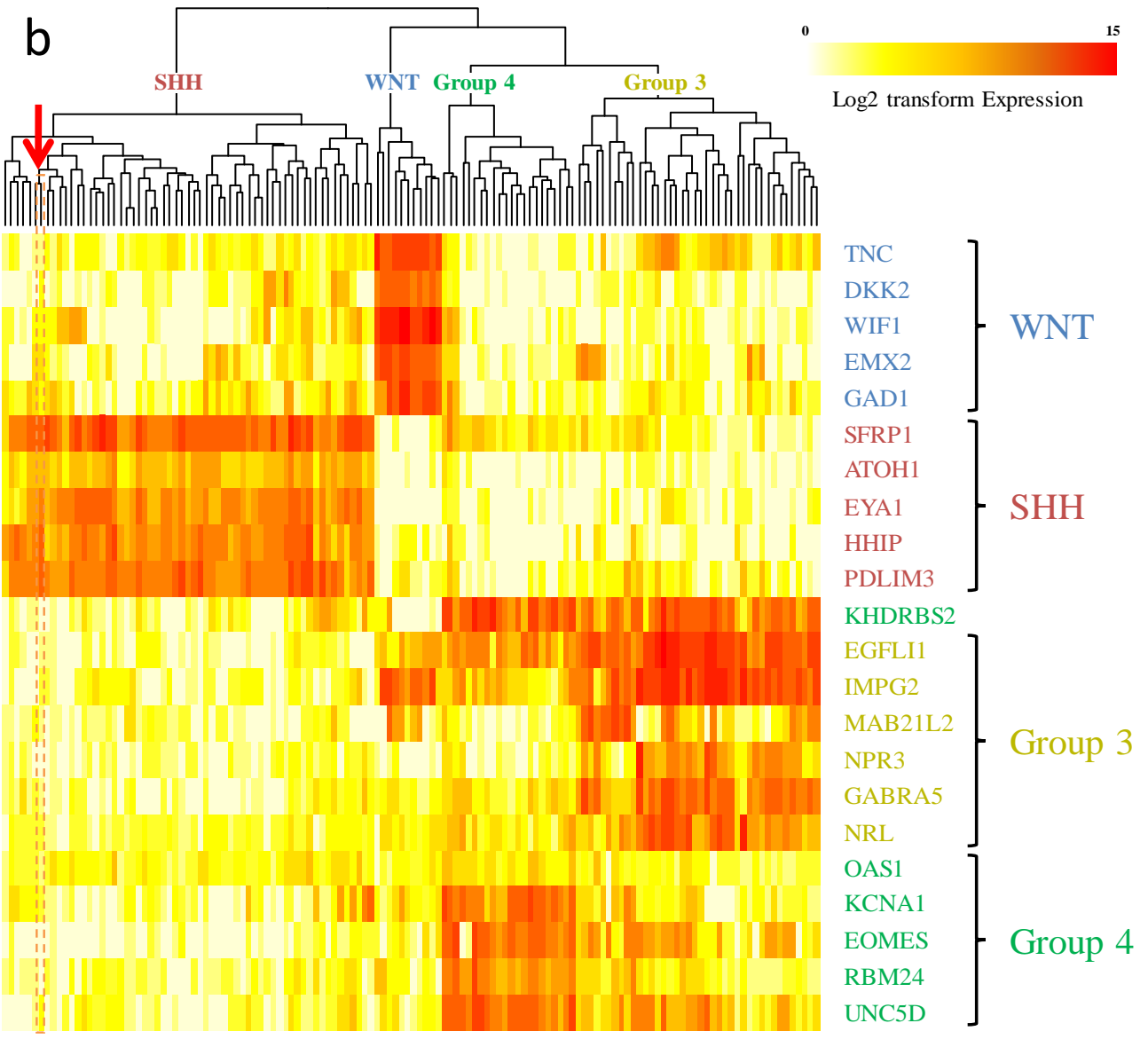

100 


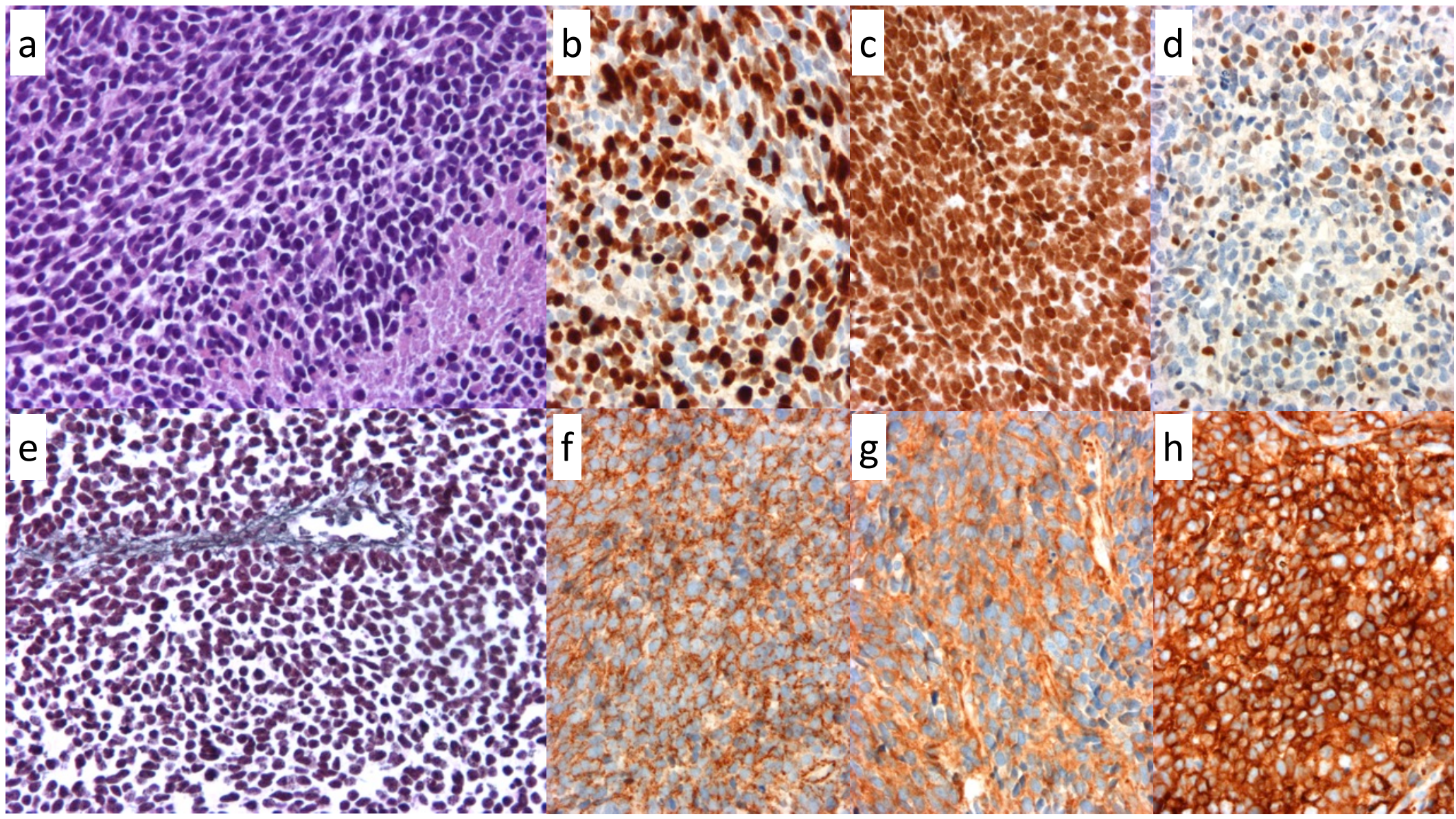




\begin{tabular}{|c|}
\hline Methylation class \\
\hline MB, SHH CHL AD \\
O IDH \\
A IDH, HG \\
A IDH \\
GBM, RTK I \\
DMG, K27 \\
MB, G3 \\
PLEX, PED B \\
EPN, RELA \\
MNG \\
MB, G4 \\
LGG, PA PF \\
GBM, RTK II \\
GBM, G34 \\
PLEX, PED A \\
EPN, PF A \\
\hline
\end{tabular}

ETMR

\section{Name}

methylation class medulloblastoma, subclass SHH A (children and adult)

methylation class IDH glioma, subclass $1 p / 19 q$ codeleted oligodendroglioma

methylation class IDH glioma, subclass high grade astrocytoma

methylation class IDH glioma, subclass astrocytoma methylation class glioblastoma, IDH wildtype, subclass RTK I methylation class diffuse midline glioma H3 K27M mutant methylation class medulloblastoma, subclass group 3 methylation class plexus tumor, subclass pediatric $B$ methylation class ependymoma, RELA fusion methylation class meningioma

methylation class medulloblastoma, subclass group 4 methylation class low grade glioma, subclass posterior fossa pilocytic astrocytoma

methylation class glioblastoma, IDH wildtype, subclass RTK II methylation class glioblastoma, IDH wildtype, H3.3 G34 mutant

methylation class plexus tumor, subclass pediatric $A$

methylation class ependymoma, posterior fossa group A methylation class embryonal tumor with multilayered rosettes
Calibrated Scores $\boldsymbol{\nabla}$

\section{C}

\begin{tabular}{|c|c|c|c|c|c|c|c|c|}
\hline Gene & Alteration & NM & DNA & Protein & Allelic Ratio $\Delta$ & Depth & SHH MB & TCF4 mutated SHH MB \\
\hline SUZ12 & Stopgain & NM_015355.3 & c. $856 C>T$ & p. $\left(\operatorname{Arg} 286^{*}\right)$ & $16,9 \%$ & $142 x$ & $0 / 123$ & $0 / 11$ \\
\hline WT1 & Nonsynonymous_SNV & NM_024426.4 & c. $835 \mathrm{G}>\mathrm{T}$ & p.(Gly279Cys) & $18,8 \%$ & $490 x$ & $0 / 123$ & $0 / 11$ \\
\hline$I D H 2$ & Nonsynonymous_SNV & NM_002168.3 & c. $514 A>G$ & p.(Arg172Gly) & $22,3 \%$ & $1234 X$ & $0 / 123$ & $0 / 11$ \\
\hline TERT & Upstream & NM_198253.2 & C. $-124 C>T$ & p.? & $46,0 \%$ & $126 X$ & $44 / 123$ & $8 / 11$ \\
\hline$B C O R$ & Nonsynonymous_SNV & NM_017745.5 & c. $5071 \mathrm{G}>\mathrm{C}$ & p.(Asp1691His) & $49,8 \%$ & $1069 x$ & $7 / 123$ & $0 / 11$ \\
\hline TCF4 & Frameshift & NM_001083962.1 & c.1241del & p.(Gly414Valfs*48) & $50,0 \%$ & $201 x$ & $11 / 123$ & $11 / 11$ \\
\hline RASA1 & Splicing_SNV & NM_022650.2 & c. $1822 A>G$ & p.(Thr608Ala) & $54,0 \%$ & $272 x$ & $0 / 123$ & $0 / 11$ \\
\hline GLI2 & Full gene amplification & - & - & - & - & - & $8 / 123$ & $0 / 11$ \\
\hline PTCH1 & WT & - & - & - & - & - & $50 / 123$ & $8 / 11$ \\
\hline$I D H 1$ & WT & - & - & - & - & - & $5 / 123$ & $1 / 11$ \\
\hline
\end{tabular}

